\title{
FTIR-microscopy as a tool for the measurement of the morphology of industrial, polymeric products
}

\author{
J.M. Chalmers* and N.J. Everall \\ ICI Technology, Wilton Research Centre, PO Box 90, Wilton, Middlesbrough, Cleveland TS90 8JE United Kingdom
}

\begin{abstract}
Many polymeric products are manufactured or fabricated into forms in which the polymer chains are oriented (aligned) to some extent. For example, fibres tend to have directional (longitudinal) stiffness imparted by polymer chains that have a preferred orientation towards or along the fibre long axis. By contrast, in many film and bottle products the macromolecular chains are typically organised to impart a balanced or nearbalanced system, that is they are biaxially oriented with respect to the product surface plane. Polarised infrared spectroscopy is a particularly useful, well established and readily practible tool for investigating polymer anisotropy. Associated with or independent of molecular orientation may be material density variations throughout a product. FTIR-microscopy is especially suited to determining and profiling localised extents of both molecular orientation and configuration. Depending on the study purpose, sample geometry, sample dimensions and sample absorption characteristics, then either a transmission or reflection, specular or internal reflection spectroscopy, technique may be employed. This article will outline briefly the principles of determining crystallinity and molecular orientation by mid-infrared techniques. It will, through case studies, illustrate applications of different FTIRmicroscopy or micro-sampling technique approaches to characterising either crystallinity or molecular orientation over a range of plastic products.
\end{abstract}

\section{Principles}

Polymer chains for many polymers may pack together in a regular arrangement to form a three-dimensional crystalline phase. These ordered phases influence the mid-infrared spectrum of the polymer, giving rise to absorption features or effects that may be directly associated with the regular conformations and their packing. For example, they may simply lead to a narrowing of an absorption band as the randomness of a system decreases, they may lead to increased intensity of a band as preferred conformations increase in concentration, or they may give rise to new bands associated with intermolecular interactions. For practical purposes, these changes may be correlated with differences in polymer crystallinity or density, through band width or band intensity measurements or by application of a multivariate data analysis technique [1].

The degree of molecular orientation of a polymer can be quantified by the second moment of the orientation distribution function, $\mathrm{P}_{200}[2,3]$. Figure 1 illustrates the relationship between $\mathrm{P}_{200}$ and the average value of $\cos ^{2} \theta$ angle, i.e $\left\langle\cos ^{2} \theta\right\rangle$, for polymer chains with respect to the draw axis for a system with uniaxial symmetry, with no preferred orientation about the draw axis. $\mathrm{P}_{200}$ may be determined from measurements of the dichroic ratio. The procedure is outlined and shown schematically in figure 2, for the situation in which the transition dipole moment lies either parallel with or perpendicular to the polymer chain axis. This highlights the need to know the transition dipole angle in order to compute $\mathrm{P}_{200}$ values from the dichroic ratio data. Polarised infrared spectra are recorded with the electric vector of the polariser alternately aligned parallel and perpendicular to the sample draw axis, and the dichroic ratio is given by the ratio $A_{\|} / A_{\perp}$. The ratio, $A_{\|} / A_{\perp}$, of the measured absorbances for a particular band represent its dichroic ratio. If the transmission dipole moment, $\mu$, associated with the particular absorbance band can be considered as lying along or perpendicular to the chain axis direction then the treatment simplifies to that shown in figure 2. The value of $\mathrm{P}_{200}$ can be shown to vary from $-1 / 2$ for perpendicular alignment through zero for random chain orientation (all $\theta$ equally likely) to unity for perfect chain alignment along the draw axis (all $\theta=0)$. It is an average over all the orientations present, and $\left\langle\cos ^{2} \theta\right\rangle$, see figures 1 and 2 , denotes the average cosine-squared of all $\theta$ angles present. A more rigorous and detailed explanation may be found in a recent tutorial publication by one (NJE) of us [4], which covers in detail the derivation of the equations for the case when the dipole moment angle lies between $0^{\circ}$ and $90^{\circ}$.

It is important to stress that the orientation distribution function is limited in its description of a system in that it does not characterise the shape of the distribution. Nevertheless $\mathrm{P}_{200}$ values are a useful tool for comparing trends in sample sets for characterising anisotropy within a sample, and investigating the mechanism of polymer network deformation. 


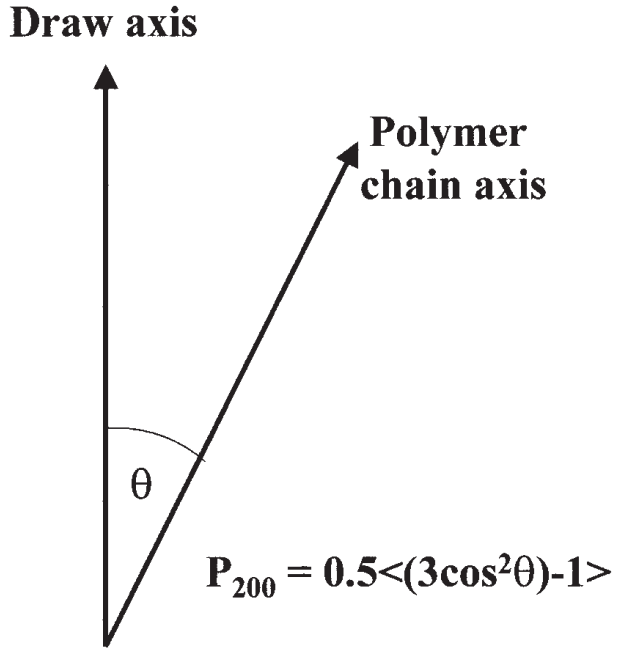

Figure 1. Illustration of the relationship between $\mathbf{P}_{200}$ and the average value of $\cos ^{2} \theta$ for polymer chains with respect to the draw axis for a system with uniaxial symmetry.

\section{Transmission}

Few manufactured polymeric products are amenable directly to mid-infrared transmission measurements of molecular orientation, excepting thin films, owing to the typical strength of their absorption bands. However, for the first case study discussed below we have been able to take advantage of the relatively weak absorption characteristics of poly(ethylene) and examine samples of ca. $0.6 \mathrm{~mm}$ thickness.

For FTIR-microscopy studies a microtomed section might seem to offer potential to provide a more favourable specimen, also enabling measurements to be made with a lateral spatial resolution approaching $10 \mu \mathrm{m} \times 10 \mu \mathrm{m}$. However, this sampling technique has a high likelihood of altering sample morphology, certainly near the surface of a cut section. We have limited experience applied to PET films and bottles and polypropylene film [5]. Our investigations showed that a small amount of orientation is induced into the microtoming direction for previously unoriented PET samples. For one way drawn films similarly small amounts of extra orientation are produced when sections are microtomed parallel to the draw direction, but very significant reorientation may be induced when sections are microtomed perpendicular to the draw direction. The technique is therefore inappropriate for measuring low levels of biaxial orientation in one-way drawn PET samples. We found however that, with care, it may be used usefully to prepare sections for comparative studies from biaxially oriented PET products [5]. For polypropylene, which has a lower $\mathrm{T}_{\mathrm{g}}$ and is less rigid than PET, we found that the procedure of room temperature microtoming to produce specimens for molecular orientation investigations was totally inappropriate, since

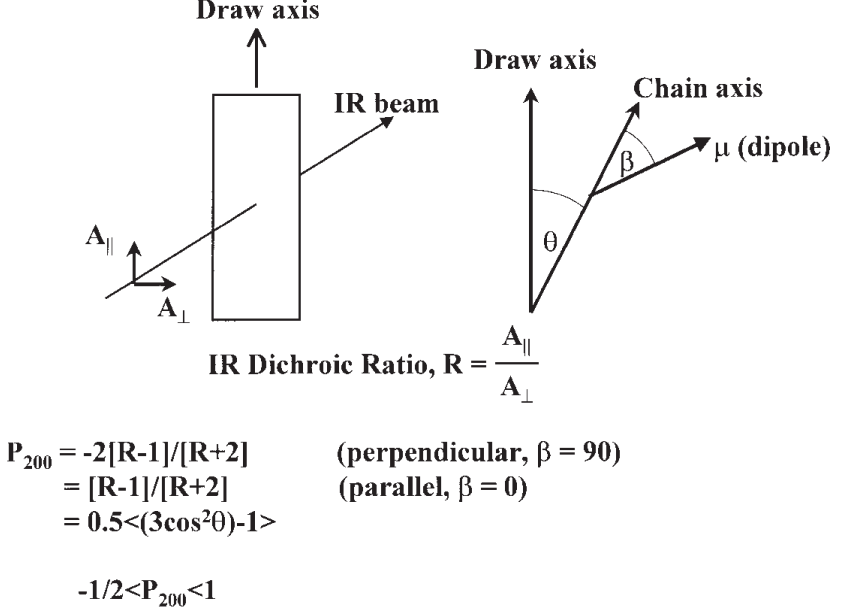

Figure 2. Illustration of the procedure for determining the dichroic ratio and $P_{200}$ from polarised infrared measurements.

significant orientation was induced in all cases. Similar observations have been made by other workers [6] in a study of the influence of microtomy on measurements of orientation by FTIR-microscopy. They concluded that sectioning by microtomy had a considerable influence on the measured orientation for the semi-crystalline polymer, polypropylene, somewhat less, but still altered, for an amorphous polymer, polycarbonate. The induced effects were both section thickness and sample preorientation dependent; for uniaxially preoriented samples they also depended on the direction and velocity of the cut [6]. Even in circumstances when it may be used, microtoming suffers from being very labour intensive and time consuming.

\section{Case study 1: Transmission FTIR-microscopy of thick-walled PE tube}

The production and physical properties (e.g. stiffness, crack resistance, electrical insulation, barrier characteristics) of poly(ethylene), PE, tubing and sheathing are very dependent upon the polymer morphology. In many applications they are critically dependent upon the polymer molecular orientation within the tubing or sheathing; in particular, the amorphous phase may be important. Vibrational spectroscopy is particularly suited to these studies since, unlike X-ray diffraction, the non-crystalline polymeric phase may be studied with relative ease. Table I lists some key absorption bands that may be used to investigate the morphology of poly(ethylenes).

For sheathing, such as that used to protect a copper wire conductor, a transflectance sampling method may provide a satisfactory direct means of comparing samples, see later 
Table I. Infrared spectrum of poly(ethylene).

\begin{tabular}{lccc}
\hline Band $\left(\mathrm{cm}^{-1}\right)$ & Phase & Transition moment & Reference \\
\hline 2016 & A + C & $0^{\circ}$ & 7 \\
1894 & C & $90^{\circ}$ & 7 \\
1368 & A & $0^{\circ}$ & 7,8 \\
1352 & A & $0^{\circ}$ & 7,8 \\
$\sim 1305$ & A & $0^{\circ}$ & 7,8 \\
1078 & A & $90^{\circ}$ & 7 \\
\hline
\end{tabular}

A, amorphous; C, crystalline.
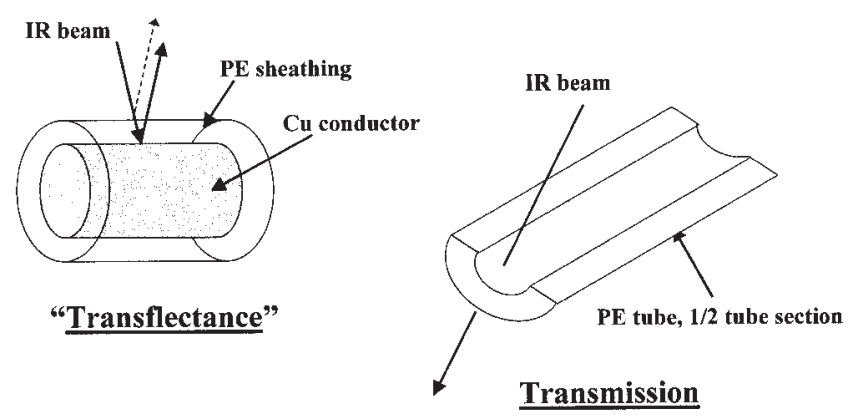

Figure 3. Schematics showing the infrared sampling procedures used for thick-walled PE tube (transmission) and PE sheathed cable ("transflectance"). and figure 3. In this approach the copper is used as the reflector and the infrared radiation is reflected from it, thereby passing through essentially twice the sheathing thickness, i.e. $2 d \cos \alpha$, where $d$ is the sheathing thickness, and $\alpha$ is the angle of incidence of the infrared beam. For thick-walled sheathing a FTIR-microscopy specular reflectance approach (in which the infrared beam is focused onto the outer surface of the poly(ethylene) sheathing) appears to offer potential, but in our experience the signalto-noise characteristics of the derived absorption index spectrum (see later) from a relatively weak absorber such as an aliphatic hydrocarbon polymer limits quantitative studies to the absorption bands of the fundamental modes of vibration $[9,10]$. Investigations undertaken using a ATR objective also failed to yield poly(ethylene) spectra of adequate signal-tonoise.

The optimum method for thick-walled poly(ethylene) tubing was to cut a section of the tube in half longitudinally, and to examine the sample in transmission normal through its wall thickness [9], see schematic in figure 3. Although this procedure maintained the integrity of the physical characteristics of sample, since the sample was examined essentially without special preparation, the specimen thickness of ca. $0.6 \mathrm{~mm}$ meant that observations were limited mostly to weaker absorption bands, particularly those in the region $2100 \mathrm{~cm}^{-1}$ to $1800 \mathrm{~cm}^{-1}$. Within this region two key bands are those at $2016 \mathrm{~cm}^{-1}$ and $1894 \mathrm{~cm}^{-1}$, which have been assigned to all-trans conformers [7]. The higher wavenumber band exists in both the amorphous and crystalline phases, and is a parallel band, in that the transition dipole moment associated with this mode of vibration lies essentially along the extended chain axis. The $1894 \mathrm{~cm}^{-1}$ has essentially perpendicular character, and only resides in crystalline regions [7]. The $1078 \mathrm{~cm}^{-1}$, with perpendicular character, is another useful indicator of amorphous phase molecular orientation.

In the case study illustrated here a primary tube was first cold drawn to differing draw ratios $(4: 1,5: 1,6: 1,7: 1)$ to induce high levels of molecular orientation. These drawn hollow tubes were then thermally annealed to relax the ori- ented polymer, by immersing the samples in water at 80 $85{ }^{\circ} \mathrm{C}$ for about 30 seconds. Examples of the polarised FTIRmicroscopy absorbance spectra recorded from transmission measurements $\left(4 \mathrm{~cm}^{-1}, 500\right.$ scans $)$ on "half-tube" specimens of these sample are shown in figure 4. The aperture used to mask the sample was $420 \mu \mathrm{m} \times 60 \mu \mathrm{m}$. Figure 5 compares the $\mathrm{P}_{200}$ values determined for the drawn and relaxed series of extended tube samples. Quantitative analysis (Fig. 5) shows that the crystalline phase is highly oriented in the drawn tubing, but relaxes slightly on annealing. The gauche conformers $\left(1078 \mathrm{~cm}^{-1}\right)$ in the amorphous phase are only slightly oriented in the drawn tube, but relax by about 30$50 \%$ on annealing, depending on draw ratio. Similar relaxations were determined from a complementary study undertaken using polarised resonance Raman spectroscopy. In this case, the process was monitored through observations made on a polyene probe that had been blended into the samples at a level of $\sim 0.5 \%$ [11]. This indicated more the changes occurring in the amorphous network as a whole, rather than simply the gauche conformers, since the linear dye (polyene) molecules, although residing in the amorphous phase, were highly oriented during drawing of the tube.

\section{Case study 2: Transmission FTIR-microscopy of microtomed sections}

As mentioned above, we have found circumstances where it was acceptable and effective in terms of the industrial purpose to undertake a particular study using polarised infrared transmission measurements on room temperature microtome prepared sections. These have been on biaxially oriented PET samples $[5,12,13]$, both thick films and soft drinks (stretch-blow-moulded) bottles. In each case our particular interest was to map variations in morphology through its thickness, perpendicular to the products' surfaces.

For example, in the tube structure of a bottle, using $120 \mu \mathrm{m} \times 120 \mu \mathrm{m}$ apertures to define the sampling area, the extent of molecular orientation was shown to decrease from near the inside surface to near the outside surface [5]. This 

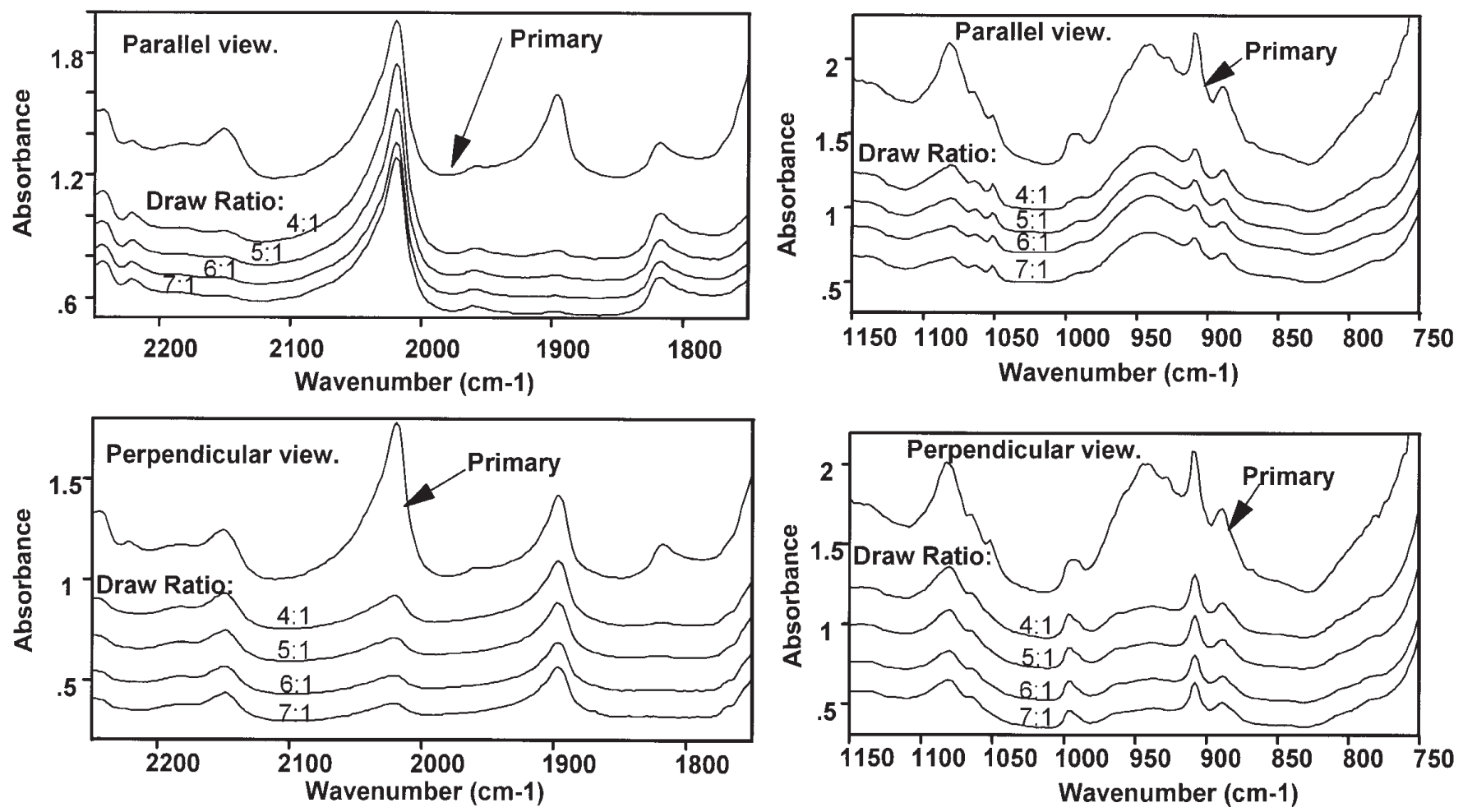

Figure 4. Polarised FTIR-microscopy spectra recorded in transmission from PE "half-tube" cold drawn specimens. The Primary sample is the original tube before the cold draw process has taken place.
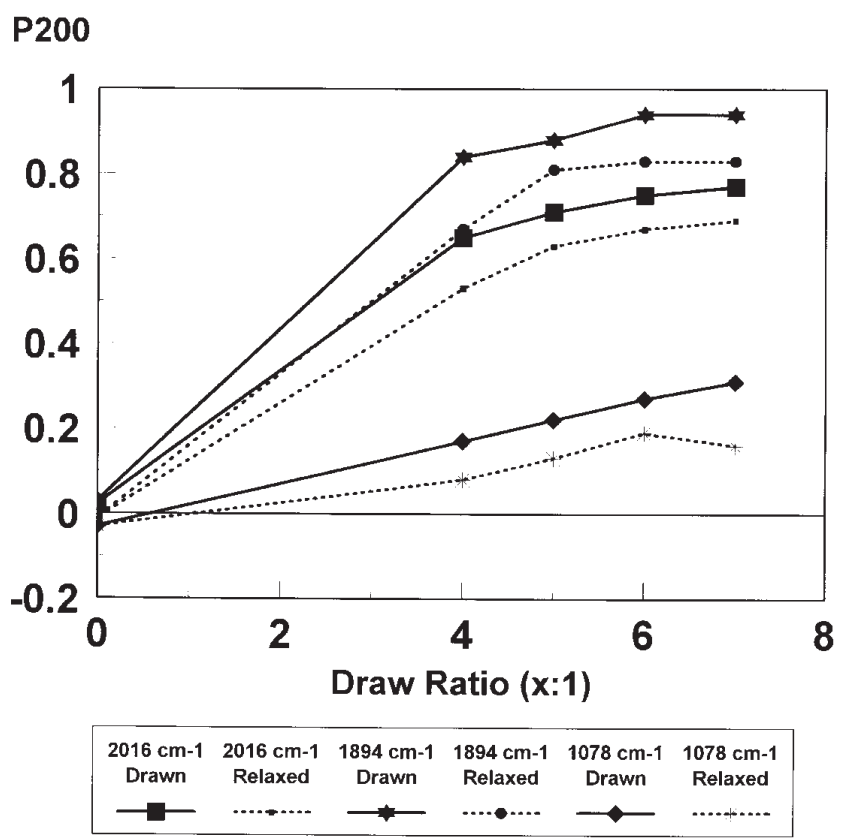

Figure 5. $P_{200}$ values computed from absorbances measured from spectra of the Primary tube, the cold drawn tubes and the cold drawn and relaxed tubes. observation was in accord with the relative extensions experienced by the bottle surfaces in the stretch-blow-moulding process. For a particular FTIR-microscopy investigation into thick (ca 350 microns) film curl the dichroic ratios of the PET absorption bands at $1018 \mathrm{~cm}^{-1}$ (parallel character) and $875 \mathrm{~cm}^{-1}$ (perpendicular character) were determined [12,13]. These were complemented by spatially resolved measurements using unpolarised infrared radiation of molecular conformation using the bands at $975 \mathrm{~cm}^{-1}$ (trans conformer) and $898 \mathrm{~cm}^{-1}$ (gauche conformer). Additionally, crystallinity was inferred from the bandwidth of the $975 \mathrm{~cm}^{-1}$. From these measurements, it was possible to deduce that underlying molecular reason for curl of the film was that the film was inhomogeneous with respect to molecular orientation through its thickness, see figure 6 . The reduced orientation towards the concave surface might indicate that the film had shrunk on this surface.

\section{Reflectance}

For many industrial purposes, a reflectance approach may offer a viable alternative to a transmission measurement, particularly when surface layer morphology is important or when surface layer characteristics may be used to imply 


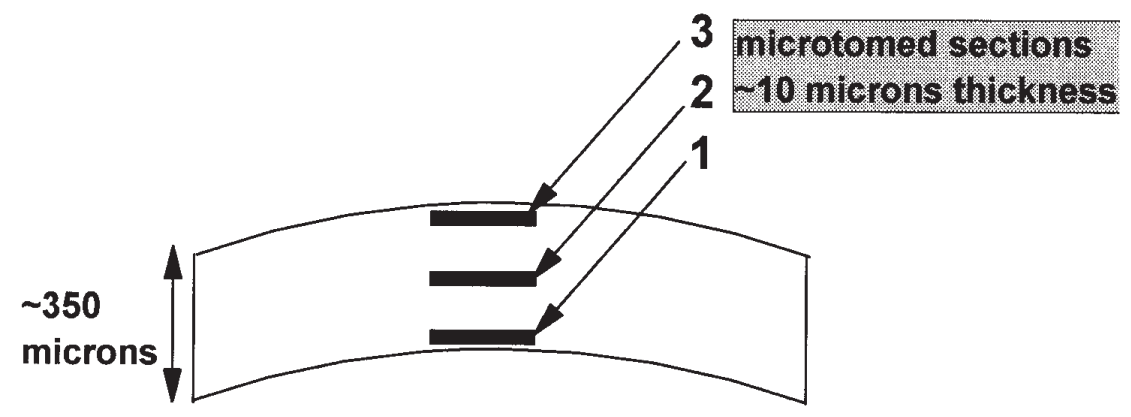

Dichroic Ratios, DR.

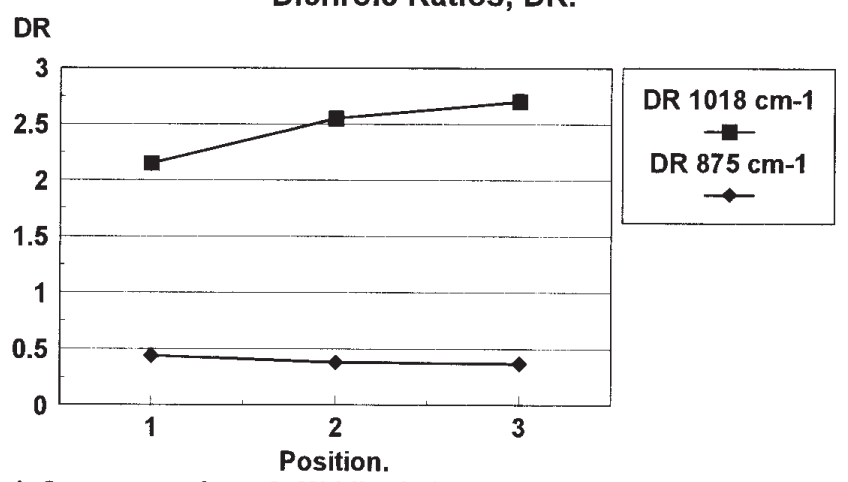

1, Concave surface; 2 , Middle; 3 , Convex surface.
$\%$ trans. \& $\%$ cryst.

$\%$ trans. \& \% cryst.

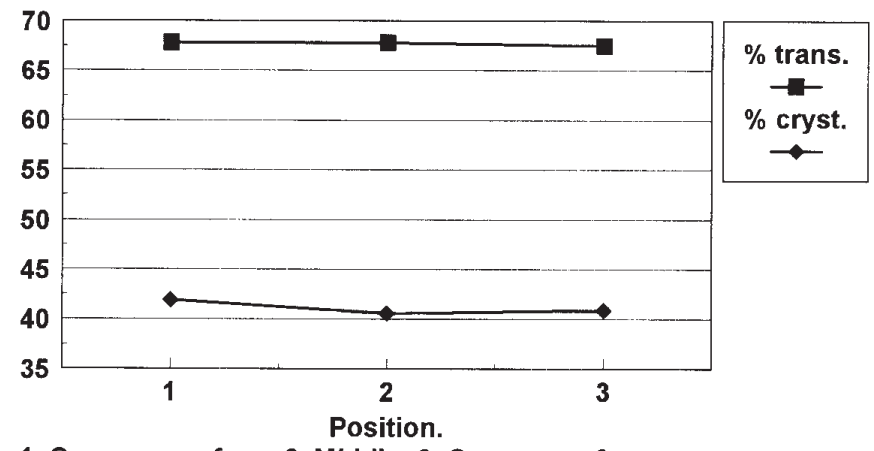

1, Concave surface; 2 , Middle; 3 , Convex surface.

Figure 6. Schematic of a microtomed cross-section from a curled PET film sample illustrating the positions measured by FTIRmiscroscopy, and profiles generated of molecular orientation, molecular conformation and crystallinity from these measurements through the thickness $(\sim 350 \mu \mathrm{m})$ of the film.

process influences on bulk product properties. It can also be advantageous for examining awkward shaped articles, such as bottles.

\section{Case study 3: Specular reflectance FTIR-microscopy of PEEK mouldings}

A front-surface external reflectance FTIR-microscopy measurement undertaken at near normal incidence is a convenient method for obtaining the absorption index spectrum from a surface layer of an "optically-thick" polymer sample. The measured specular reflectance spectrum is a convolution of dispersion in the sample refractive index with the absorption profile. These may be separated to yield the more analytically useful absorption index and refractive index spectra by subjecting the recorded specular refelectance spectrum to the Kramers-Kronig (KK) algorithm. This process is shown schematically in figure 7.

The measurement requires that the sample area examined is essentially optically flat and free from surface contamination. The sample must be of a sufficient thickness or chemical constitution such that it is "optically thick", that is no radiation other than that directly specularly reflected from its front surface should impinge on the FTIR detector. Radiation entering the sample must not be returned by reflectance from another surface or interface, or through diffuse reflectance processes. The raw data must be ratioed against the spectrum of a "perfect" reflector, often a polished gold surface, before computing the KK transform. Because one is limited by the signal-to-noise available within the generated absorption index spectrum, the approach favours the more strongly absorbing polymers, such as aromatic polymers.

Figure 8(a) shows the absorption index spectra obtained from measurements of the specular reflectance spectra from a series of poly(aryl ether ether ketone), PEEK, plaques of differing crystallinities $[10,14]$. The spectra show progressive changes in relative absorption band intensities that may be correlated directly with the differing crystallinities. Figure 8(b) is a plot of such a correlation. The $4 \mathrm{~cm}^{-1}$ resolution spectra, 100 scans, were recorded on a Nicolet 850 FTIR spectrometer interfaced to a Nic-Plan microscope, using an aperture size of $360 \mu \mathrm{m} \times 360 \mu \mathrm{m}$. The PEEK plaques were of nominal thickness $0.6 \mathrm{~mm}$. 


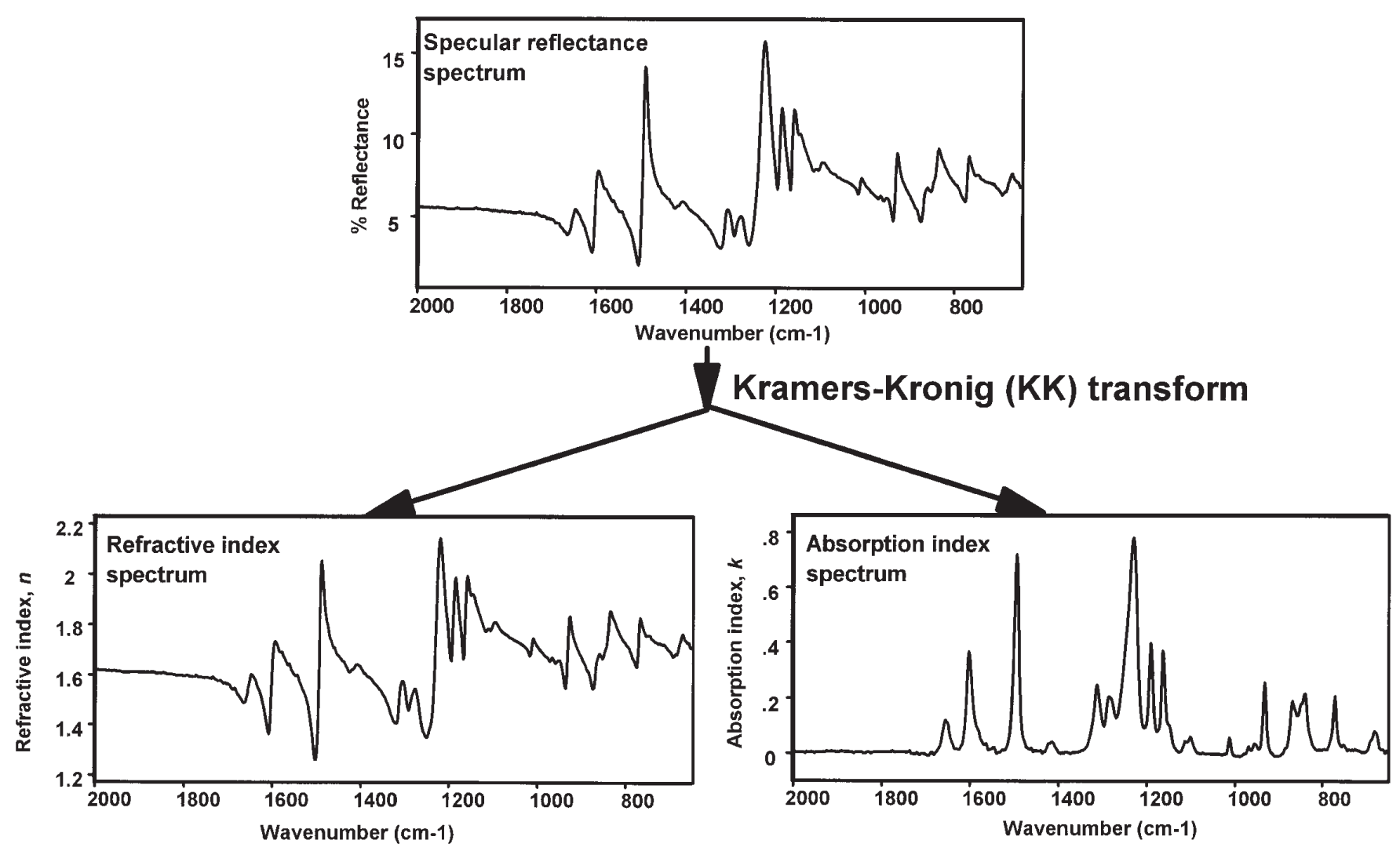

Figure 7. Schematic illustrating application of the Kramers-Kronig transform to a recorded specular reflectance spectrum.

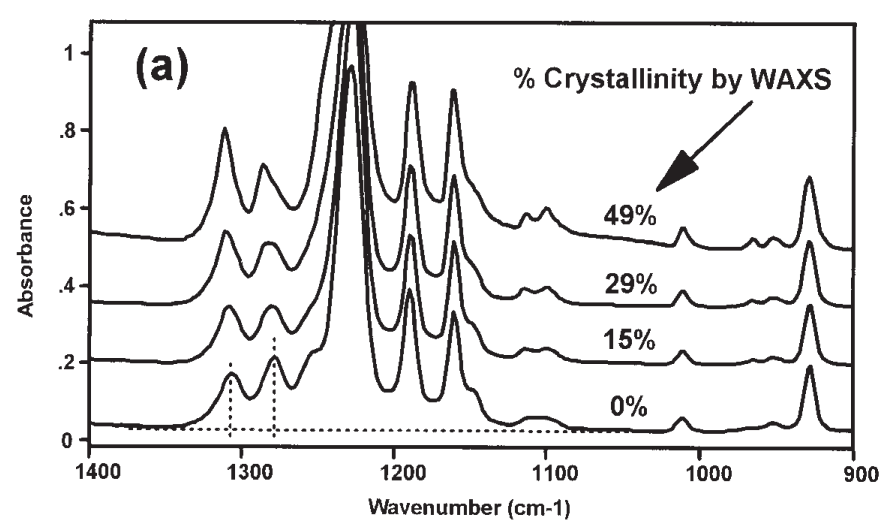

Absorption index intensity ratio: $1305 \mathrm{~cm}-1 / 1280 \mathrm{~cm}-1$

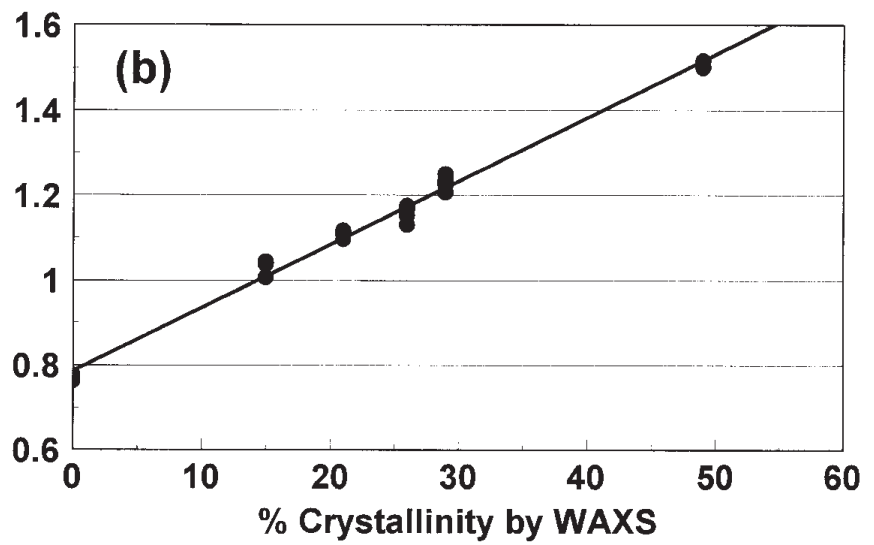

Figure 8. (a) Absorption index spectra generated from application of the Kramers-Kronig transform to the recorded specular reflectance spectra for a series of PEEK plaques of differing crystallinities, (b) Plot of absorption index band ratio $\left(1305 \mathrm{~cm}^{-1} / 1280 \mathrm{~cm}^{-1}\right)$ against WAXS determined \% crystallinity for the series of PEEK plaques. 
Reference data like these may then provide the base from which to extend measurements to manufactured articles. Figures 9(a) and 9(b) show a crystallinity profiles produced from FTIR-microscopy point mapping a section of the surface of the product shown in figure 9(c).

\section{Case study 4: Specular reflectance FTIR-microscopy of PET films}

In the case study above, we illustrated how FTIRmicroscopy specular reflectance measurements may be used to map localised lateral variations in surface layer crystallinity on polymer articles. Here, we discuss applications to measure molecular orientation in poly(ethylene terephthalate), PET, products [15]. In order to minimise transflectance components, so that a sample may be considered as "optically thick", the approach is limited to samples of thickness $50 \mu \mathrm{m}$ or greater. In a development study [15], a range of uniaxially drawn films was analysed. These had forward draw ratios varying from 1 to 3.5 . Their thickness ranged from $420 \mu \mathrm{m}$ to $120 \mu \mathrm{m}$ according to draw ratio. (Note: for this case the microscope simply provided a convenient sampling tool; it was not used to profile orientation on the micro-scale).

The FTIR-microscope aperture was set at about $90 \mu \mathrm{m} \times 480 \mu \mathrm{m}$ and a film sample examined with infrared radiation that was nominally polarised either parallel or perpendicular to the draw direction of the film. Prior to quantitative analysis, the "first-derivative" like specular reflectance spectra were pre-processed. The approaches adopted were the more conventional application of the Kramers-Kronig algorithm to extricate the absorption index spectra and differentiation to yield a "second-derivative" appearance spectrum [15]. In subsequent analyses, both approaches gave similar results for calculated orientation parameters. The determinations were based on measurements of the $1019 \mathrm{~cm}^{-1}$ ring stretching band of PET, see figure 10(a). To correct for non-ideal influences such as polarisation-dependent surface texture effects on sample reflectivity, all band intensities within a spectrum were (a)

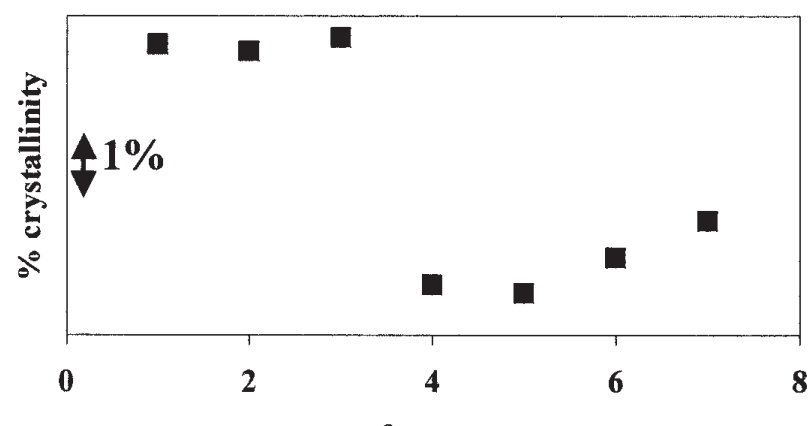

cm. from centre

(b)

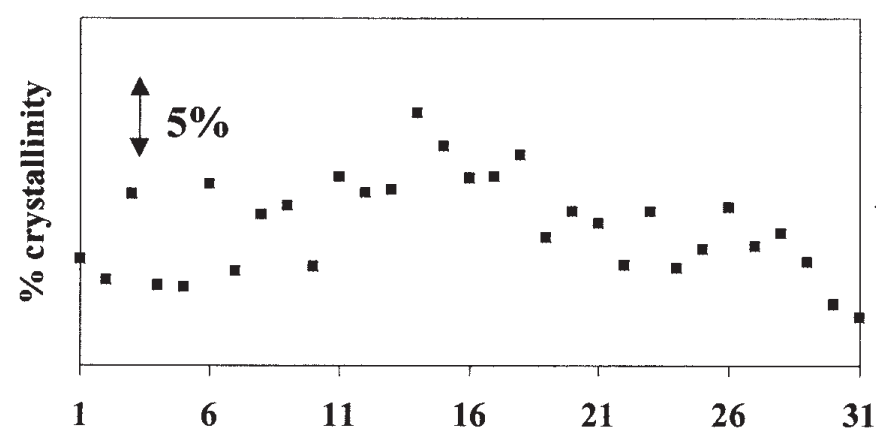

sampling position $(\mathrm{cm}$.) along length axis

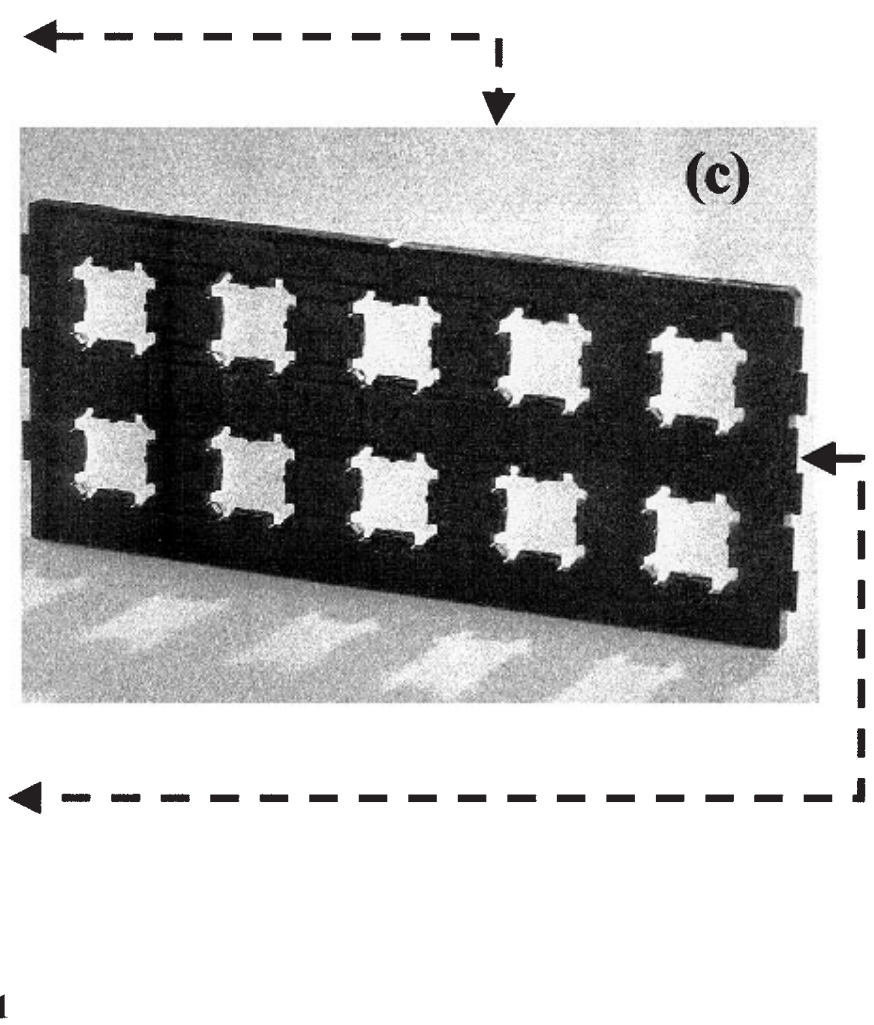

Figure 9. PEEK \% crystallinity profiles, (a) along a short arm axis and (b) along the length axis, determined from specular reflectance FTIR-microscopy measurements of the PEEK moulding pictured in (c). 


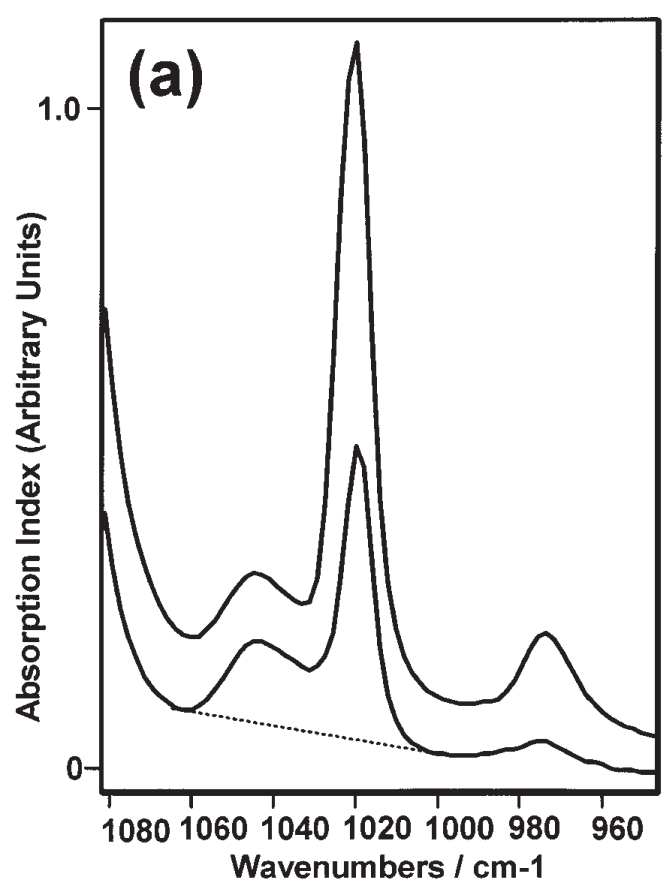

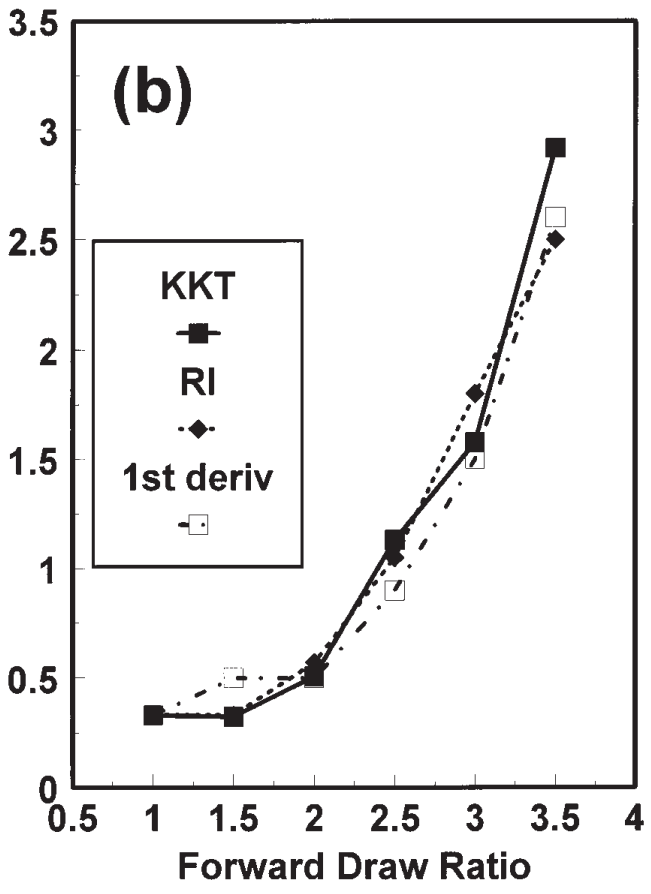

Figure 10. (a) KK transformed spectra of $3.5: 1$ uniaxially drawn PET sample: the top spectrum was generated from a specular reflectance measurement made using radiation polarised parallel to the draw direction; the bottom spectrum was generated from a specular reflectance measurement made using radiation polarised perpendicular to the draw direction. (b) Comparison of $P_{200}$ values, see text for details. normalised relative to that of the $1410 \mathrm{~cm}^{-1}$ ring mode, which is a non-dichroic band [15-17]. Figure 10(b) compares the infrared specular reflectance derived $\mathrm{P}_{200}$ values with those determined from refractive index measurements; these data sets are seen to be in close agreement.

This FTIR-microscopy reflectance approach has an advantage in that only small amounts of a sample are required and that they need be flat only on the sub-millimetre scale [15]. It does however require a thick sample in order that an esentially pure specular reflectance spectrum may be recorded and "transflectance" spectral components are eliminated. Also, it is probably limited in application to strongly absorbing polymers, and benefits from the normalisation procedure invoked to appease much of the non-ideal practical circumstances. However, the most serious defect of this approach is that quantitative $\mathrm{P}_{200}$ values can only be computed for uniaxial samples. Biaxial samples require either near-grazing reflectivity measurements or polarised ATR investigations (see case study 6 below).

\section{Case study 5: Transflectance FTIR-microscopy of PE sheathed wire}

The polarised FTIR microreflectance spectra shown in figure 11 were recorded from two cable samples of copper wires sheathed in high-density poly(ethylene), HDPE. They were recorded at $4 \mathrm{~cm}^{-1}$ resolution using the transflectance method [18], see figure 3. The $\mathrm{Cu}$ conductor was $2.5 \mathrm{~mm}$ thick; the HDPE sheathing was $150 \mu \mathrm{m}$ thick. The molecular anisotropy in each is evident from a comparison between the pairs of parallel and perpendicular polarised absorbance spectra, recorded using infrared radiation alternately polarised parallel and perpendicular to the cable lengths. The two cables were manufactured at different line speeds, and it is evident from comparing the relative intensities of bands between these spectra that the line speed influences the degree of molecular orientation of the HDPE. Simple comparative observations like these can be important, since the performance of plastic coated wires and cables can be affected by the molecular ordering of the polymer sheathing.

It should be remembered that spectra recorded in transflectance are a composite of the attenuated radiation that has traversed through the sample to a pathlength equivalent to $2 d \cos \alpha$, see above, and a weaker specular reflectance component, illustrated in figure 3 by the dashed line. This front surface reflection contribution will distort band intensities, but has minimal effect on bands of low absorptivity, as is the case here, and its effect is therefore probably negligible. 

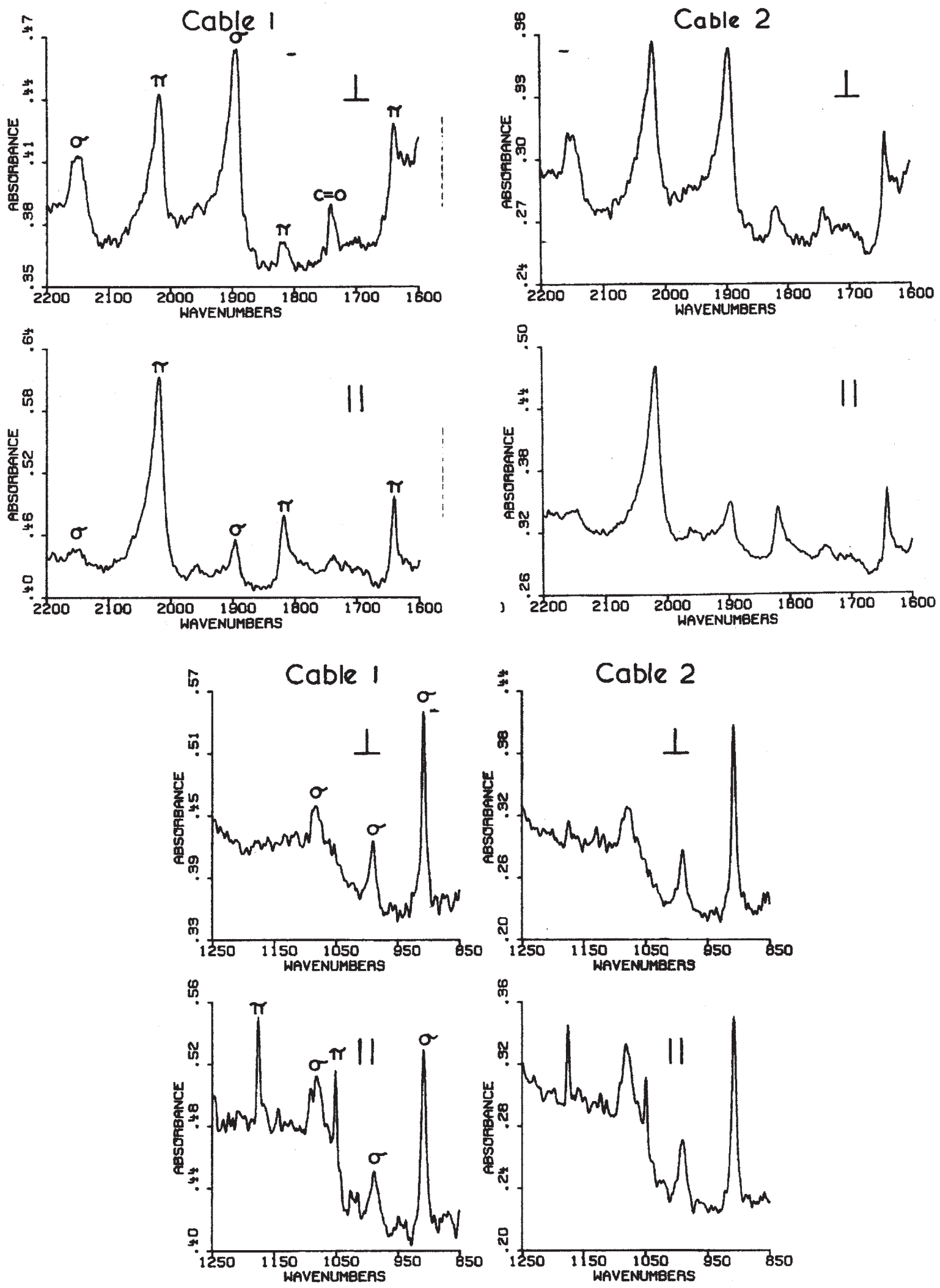

Figure 11. Polarised "transflectance" micro-FTIR measurements made on two samples, manufactured at different line speeds, of poly(ethylene) sheathed copper conductors. 


\section{Case study 6: FTIR ATR-microsampling of PET films}

Although FTIR-microscopy with an ATR objective would appear clearly to offer potential for spatially-resolved determinations of the molecular orientation characteristics of a polymer article, we are not aware of any publications to date demonstrating this. In early attempts we made with one commercial system we encountered considerable practical difficulties. The contact surface of ZnSe ATR elements rapidly became scratched, and consequently scrambled the polarisation and became unfit for the purpose. With harder Ge prisms we were unable to reproducibly achieve sufficient intimacy of surface contact to reproduce spectra of the necessary signal-to-noise. [However, newer designs of ATR objectives may well facilitate this measurement, but as yet we have no experience with these]. As an alternative, surface layer measurements of molecular orientation were undertaken in our laboratory using a robust, single-reflection accessory [19]. The contact surface of this diamond, $45^{\circ}$ incidence angle, internal-reflection element was sufficient for our investigations, since in the investigations being undertaken we were not driven by the need for very high spatial profiling of molecular orientation.

The localised surface area studies were made in our laboratory using a "Golden-Gate ${ }^{\mathrm{TM} "}$ (Graseby Specac Ltd., Orpington, Kent, U.K.) accessory. The diamond element contact area of this unit is about $4 \mathrm{~mm}^{2}$. Reproducible contact pressure against the sample surface is made through a sapphire anvil tightened with a torque wrench, see schematic in figure 12. (More recently, other commercial low surface contact area ATR accessories have become available, for example: MIRacle ${ }^{\mathrm{TM}}$ from Pike Technologies, Inc., Madison, WI, USA ; Thunderdome ${ }^{\mathrm{TM}}$ from Spectra-Tech, Shelton, CT, USA; DuraSamplIRT ${ }^{\mathrm{TM}}$ form ASI Applied Systems, SensIR Technologies, Danbury, CT, USA). The key to the success with the method is that prior to calculating dichroic ratios, absorption band intensities were normalised with respect to the non-dichroic PET band at $1410 \mathrm{~cm}^{-1}$ [19], see figure 13 . With this approach the need to maintain identical contact pressures and sampling areas is eliminated, as is the need to know the refractive indices for the polymer concerned, which in turn are orientation dependent!). (Reliable and reproducible sampling following specimen removal, rotating and reclamping has been a major drawback for ATR dichroic measurements). The theory underlying calculating orientation parameters from dichroic ATR data is outside the scope of this article, but has been set out by one of us in reference 19. This publication also considers the effects of refractive index anisotropy and dispersion on the measured values.

For the case study discussed here, a set of uniaxially drawn PET films were analysed. They covered the range $\times 1$ to $\times 3.5$ draw ratio, and were the same sample set as used for the specular reflectance study discussed previously in case study 4 . The results from this investigation are shown

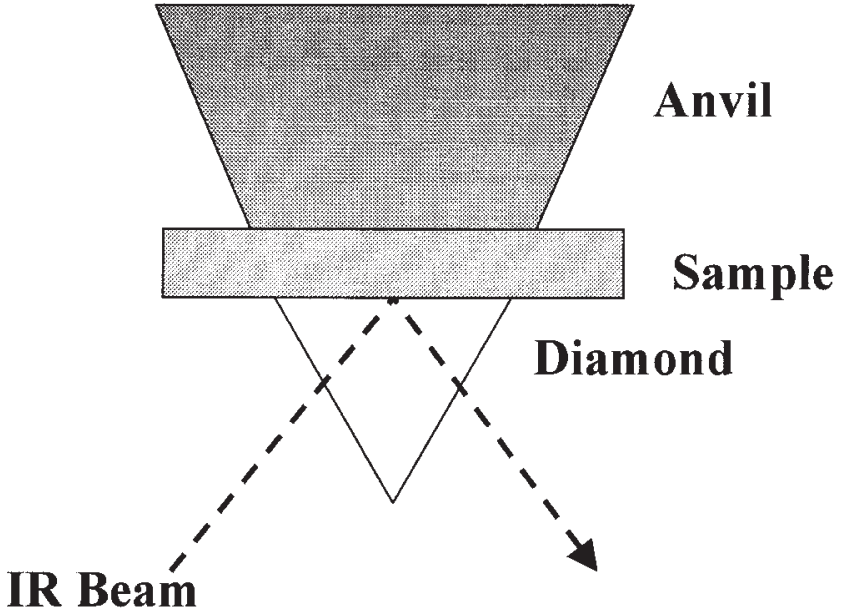

Figure 12. Schematic of "Golden-Gate ${ }^{\mathrm{TM}}$ " diamond ATR accessory. A single bounce at $45^{\circ}$ incidence is employed.

in figures 14 and 15 . The specular refelectance data presented for comparison in figure 14 differ from those shown in figure 10(b). Although they were from the same sample set, in figure 14 the data were generated from measurements taken from the inner surface of a film roll, whereas those of figure 10(b) were from studies on the outer surface. This exemplifies that many commercial films may, through their process of manufacture, exhibit anisotropy in their morphology through their thickness, and methods for profiling this have importance. We have suggested above (see case study 2) that in some circumstances examining a micro-

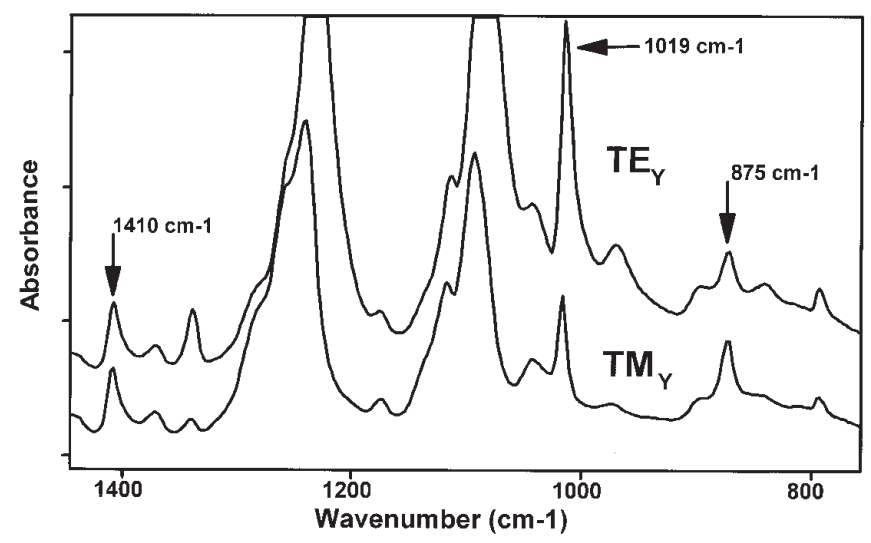

Figure 13. Spectra recorded using the Golden-Gate ${ }^{\mathrm{TM}}$ ATR accessory from $\times 3.5: 1$ uniaxially drawn PET film sample with the draw axis perpendicular to the plane of incidence of the infrared radiation. $T E_{Y}$ and $T M_{Y}$ refer to the electric vector of the polarised infrared radiation being perpendicular to or in the plane of incidence respectively, see reference 19 for full details. 
tomed section might prevail. Other potential techniques are confocal Raman microspectroscopy, step-scan FTIR photoacoustic spectroscopy and FTIR ATR microscopy using a range of different internal reflection elements. For example, polarised confocal Raman has been applied to this same sample set to measure orientation and crystallinity gradients through the film thickness $[20,21]$. None is a panacea; each has its limitations and drawbacks. Neither of the FTIR techniques will probe fully thick, strongly absorbing samples; ATR information will also be limited to surface layers extending to at best a few microns. Even if the Raman study were undertaken along a cut edge one is still subject to sectioning artefacts.

In the specular reflectance investigation (case study 4) for which the incident and reflected radiation were near-normal we were only able to access quantitative $\mathrm{P}_{200}$ information related to the extension and transverse directions, referred to as the draw or machine direction, MD, and transverse direction, TD, respectively. The great benefit of the ATR measurements is that electric field components may be specified for all three orthogonal directions [19], so additionally, dichroic data relating to the thickness direction may be determined. This is exampled in figure 15. This shows averaged squared direction cosines for the angles between the polymer chain (y) and the MD (Y), TD (X) and thickness (Z) directions. A detailed description of this comparative study is given in reference 19 .

\section{Summary}

In this article, we have described briefly several examples of the application of FTIR-microscopy techniques to investigate physico-chemical properties of polymeric products. These studies were made in the context of fit for purpose industrial problem-solving and characterisations. Although each sampling approach has limitations, the importance and value of the comparative information they supply is hopefully explicit. We have found they can provide unique insights into localised properties of polymer articles, and can have significant cost benefits for product development. Although not considered above, the application of Raman microscopy in tandem to the same problems enables even more detailed morphological characterisation, and if at all possible both approaches will normally be applied in our laboratory.

\section{Acknowledgements}

We wish to thank many ICI colleagues who have contributed to the work reviewed here, most of whom are named as coauthors with us in the references to this article. We are also indebted to ICI for permission to publish this paper.

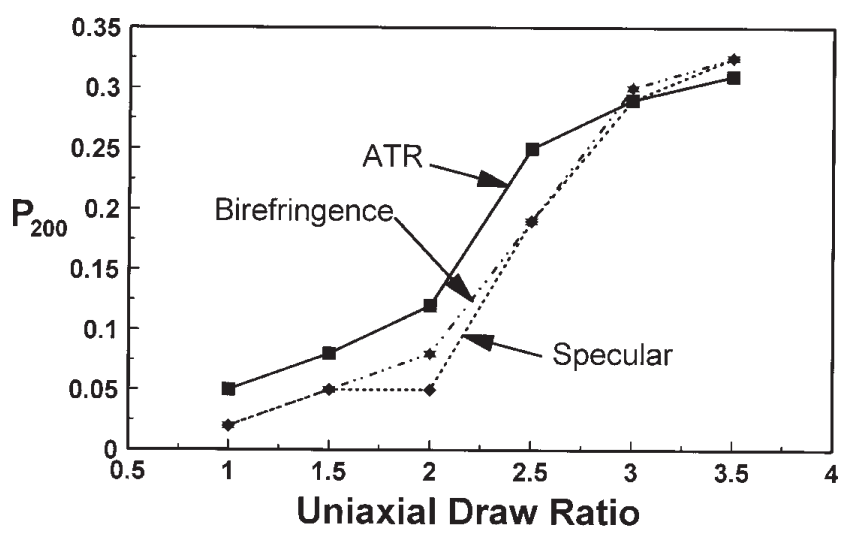

Figure 14. Comparison of $\mathbf{P}_{200}$ values as a function of draw ratio for the same PET film samples analysed by polarised ATR and specular reflectance FTIR-dichroism, and birefringence measurements.

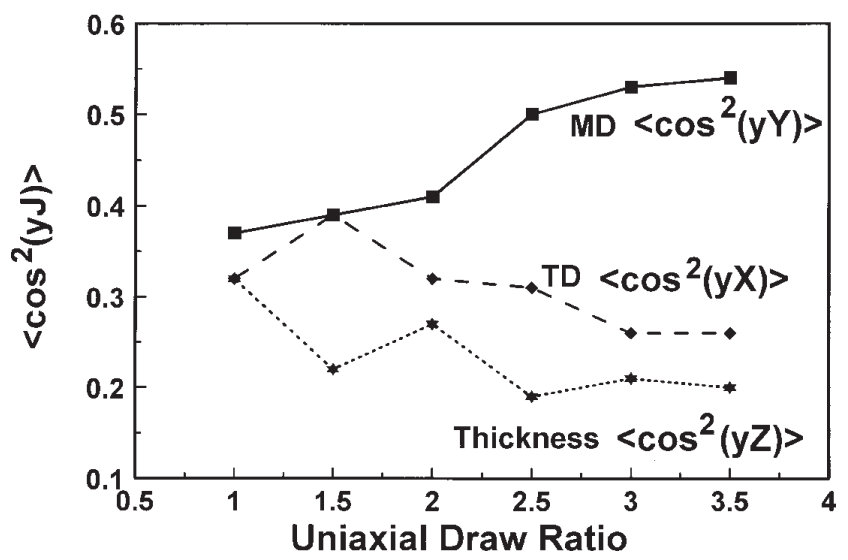

Figure 15. Comparison of the averaged squared direction cosines for the angles between the polymer chain (y) and the MD (Y), TD (X) and thickness (Z) axes computed from the ATR dichroism measurements made on the uniaxially drawn PET film sample set.

\section{References}

1. Chalmers, J.M.; Everall, N.J. in Polymer Characterisation (Hunt, B.J.; James, M.I., Eds.); Blackie Academic: Glasgow, 1993, pp 69-114.

2. Bower, D.I. in Structure and Properties of Oriented Polymers (Ward I.M., Ed.); Chapman \& Hall: London, 1997, pp 181233.

3. Jarvis, D.A.; Hutchinson, I.J.; Bower, D.I.; Ward, I.M. Polymer 1980, 21, 41-54.

4. Everall, N. Internet J. Vib. Spect. [www.ijvs.com] 1999, 3, 2. 
5. Chalmers, J.M.; Croot, L.; Eaves, J.G.; Everall, N.; Gaskin, W.F.; Lumsdon, J.; Moore, N. Spectros, Int. J. 1990, 8, 13-42.

6. Lundberg, L.; Sjönell, Y.; Stenberg, B.; Tersejius, B.; Jansson, J.-F. Polymer Testing 1994, 13, 441-459.

7. Wedgewood, A.R.; Seferis, J.C. Pure \& Appl. Chem. 1993, 55, 873-892.

8. Agosti, G.; Zerbi, G.; Ward, I.M. Polymer 1992, 33, 42194229.

9. Chalmers, J.M.; Everall, N.J.; Local A. Microbeam Analysis 1995, Proc. 29 $9^{\text {th }}$ Ann. Conf. Microbeam Anal. Soc., Breckenridge, Colorado, August 6-11, 1995; VCH Publishers, Inc.: New York, pp 123-124.

10. Chalmers, J.M.; Everall, N.J.; Ellison, S. Micron 1996, 27, 315-328.

11. Everall, N.; Chalmers, J.; Mills, P. Appl. Spectrosc. 1996, 50, 1229-1234.

12. Chalmers, J.M.; Everall, N.J. Macromol. Symp. 1995, 94, 3349.
13. Chalmers, J.M.; Dent, G. Industrial Analysis with Vibrational Spectroscopy, The Royal Society of Chemistry: Cambridge, UK, pp 1997.

14. Chalmers, J.M.; Everall, N.J.; Hewitson, K.; Chesters, M. A.; Pearson, M.; Grady, A.; Ruzicka, Analyst 1998, 123, 579-586.

15. Everall, N.; Chalmers, J.M.; Local, A.; Allen, S. Vib. Spectrosc. 1996, 10, 253-259.

16. Guevremont, J.; Ajji, A.; Cole, K.C.; Dumolin, M.M. Polymer 1995, 36, 3385-3392.

17. Cole, K.C.; Guevremont, J.; Ajji, A.; Dumolin, M.M. Appl. Spectrosc. 1994, 48, 1513-1521.

18. Chalmers, J.M.; Mackenzie, M.W. in Advances in Applied Fourier Transform Infrared Spectroscopy (Mackenzie M.W. Ed.); John Wiley \& Sons: Chichester, 1988, pp 105-188.

19. Everall, N.J.; Bibby, A. Appl. Spectrosc. 1997, 51, 1083-1091.

20. Everall, N.J. Appl. Spectrosc. 1998, 52, 1498-1504.

21. Everall, N.J.; Davis, K.; Owen, H.; Pelletier, M.J.; Slater, J. Appl. Spectrosc. 1996, 50, 388-393. 